\title{
Correlated noise unfolding on a Hadronic Calorimeter
}

\author{
M. C. N. Fiolhais on behalf of the ATLAS Tile Calorimeter Group \\ Laboratório de Instrumentação e Física Experimental de Partículas \\ Departamento de Física da Universidade de Coimbra \\ 3004-516 Coimbra, Portugal \\ Email: miguel.fiolhais@cern.ch
}

\begin{abstract}
The correlated noise component of TileCal, the barrel hadronic calorimeter of the ATLAS experiment at the Large Hadron Collider, is studied and an algorithm is used to parametrize and unfold it from the response of the photomultipliers. It is shown that the correlated noise component can be significantly reduced and mostly removed not only for pedestal runs, but also in the presence of physics signals like minimum bias events in $900 \mathrm{GeV}$ collisions and $7 \mathrm{TeV}$ simulated top quark pair production events.
\end{abstract}

\section{INTRODUCTION}

The ATLAS detector [1] is a general purpose detector designed to explore the physics potential of the Large Hadron Collider (LHC). ATLAS consists of several layers, each with a specific purpose on the reconstruction of particles. The main detector components are the Inner Tracker, the Electromagnetic and Hadronic Calorimeters and the Muon Spectrometer. The Tracker and the Muon Spectrometer are embedded in two powerful Magnet Systems. The central Hadronic Calorimeter (TileCal) [2], is composed by steel as the absorber and scintillating tiles as the active material.

The Tile Calorimeter consists of one central barrel (separated in LBA and LBC) and two extended barrels (EBA and EBC) composed of 64 wedges, also known as modules. The light produced by the particles that cross the TileCal tiles is collected at the two edges of the scintillating tiles by wavelength shifting (WLS) fibers which are bundled together to form cells with three sampling depths (A,BC and D) and with a transverse granularity of $\Delta \eta \times \Delta \phi=0.1 \times 0.1$ $(0.1 \times 0.2$ for the last layer $)$. Every cell is readout from both sides by different channels in order to provide redundancy. The channels reading out a wedge are grouped in assemblies (drawers) of 48 readout units hosted inside the girder at the outer radius of the calorimeter. The signal pulse produced by each photomultiplier is shaped, amplified with two gain factors (low gain and high gain), whose nominal ratio is 1:64, by fast and low-noise front-end electronics. The amplified signals are sampled and digitized at $40 \mathrm{MHz}$ by two 10-bit Analog to Digital Converters. During this process undesirable signal interference effects might occur as it has been reported in a previous study [3]. This will result in a correlated noise pattern between different channels which may have a negative impact on the TileCal performance. Finally, digitized samples - high-gain or low-gain depending on the signal amplitude, are sent via optical links to off-detector back-end electronics. The correlated noise seems to manifest itself in regions of 6 or 12 channels, which is the extent of the digitizers and motherboards cards, respectively. There are "sensitive" areas in the drawer front-end (FE) assembly where the electromagnetic interference between the Low Voltage Power Supply (LVPS) and FE is pronounced, i.e. the area close to the LVPS (channels $>42$ ) and the border between the two sub-assemblies (channels around 24). This effect is enhanced by the fact that switching DC-DC converters are used as LVPS.

\section{TileCal Correlated Noise}

The correlated noise in TileCal plays an important role in the pedestal fluctuations' degradation. To address the problem, it is desirable to consider a general approach based on first principles which does not depend on the specific source of the problem. The approach presented assumes that the observed noise measurement $\left(x_{i}\right)$ in a particular channel $i$ of the TileCal module, is a combination of a genuine intrinsic noise component $\left(\beta_{i}\right)$ plus a contribution which depends on the response of all readout channels in the module as a whole and it is probably dominated by the closest neighbors. The simplest approach to describe the noise measurement in channel $i$ is then to consider $x_{i}$ as being a linear combination between the intrinsic noise component $\left(\beta_{i}\right)$ and a weighted sum of the signals of all the other readout channels $(N)$ in the module i.e.,

$$
x_{i}=\beta_{i}+\sum_{j \neq i}^{N} \alpha_{i, j} x_{j} .
$$

where $x_{i}$ and $x_{j}$ are the noise signals for channels $i$ and $j$ respectively. These signals refer to the pulse amplitudes reconstructed by the non-iterative Optimal Filter method [4], [5] using the 7 digitized measurements of the pulse. The $\alpha_{i, j}$ unknown parameters ensure measurements from other readout channels are taken into account with different weights. Given the fact that pedestal subtracted signals around zero are used, the $\beta_{i}$ values are expected to be zero. For each channel, the measured noise can be compared with the model above using 
the usual $\chi^{2}$ method,

$$
\chi_{i}^{2}=\sum_{\text {Events }} \frac{\left[x_{i}-\left(\beta_{i}+\sum_{k \neq i}^{N} \alpha_{i, k} x_{k}\right)\right]^{2}}{\sigma_{i}^{2}},
$$

which can be minimized (individualy for each channel) with respect to each one of the $\alpha_{i, j}$ and $\beta_{i}$ of the model,

$$
\frac{\partial \chi_{i}^{2}}{\partial \alpha_{i, 1}}=\frac{\partial \chi_{i}^{2}}{\partial \alpha_{i, 2}}=\ldots=\frac{\partial \chi_{i}^{2}}{\partial \alpha_{i, N}}=\frac{\partial \chi_{i}^{2}}{\partial \beta_{i}}=0
$$

Assuming that the noise correlations are the same independently of the signal amplitude or, in other words, that no cross talk is present, the noise correlations information (embedded in the $\alpha$ matrix) can be extracted from the first out of seven digitized samples and the $\alpha$ matrix itself:

$$
\left(\begin{array}{cccc}
0 & \alpha_{1,2} & \ldots & \alpha_{1, N} \\
\alpha_{2,1} & 0 & \ldots & \alpha_{2, N} \\
\ldots & \ldots & \ldots & \ldots \\
\alpha_{N, 1} & \alpha_{N, 2} & \ldots & 0
\end{array}\right)
$$

The values of the $\alpha_{i j}$ and of the offset $\beta_{i}$ are obtained with the minimization procedure. The reconstruction of the signal in channel $i\left(s_{i}^{r e c}\right)$ is performed by removing the offset evaluated during the minimization procedure $\beta_{i}$ and by applying the $\alpha$ matrix to the measured values of all the other channels of the module according to,

$$
s_{i}^{r e c}=s_{i}-\left(\alpha_{i, 1} x_{1}+\alpha_{i, 2} x_{2}+\ldots+\beta_{i}+\ldots+\alpha_{i, N} x_{N}\right)(4)
$$

If the method provides a good description of the correlations in the noise pattern, one may expect that the noise distribution will be narrower after correcting any undesirable effects, closer to the intrinsic noise distribution. The level of noise correlation was studied using 10,000 events from a bi-gain pedestal run during 2009 data taking. In Figure 1 (top), correlations between channels are clearly visible in high-gain for the TileCal module LBA9. Regions of high and low correlation values are visible reflecting the configuration of the TileCal hardware with clear clusters of neighbor channels determining the signal responses, the behavior in magnitude and shape is typical for TileCal drawers. These correlations were significantly reduced after applying the correlated noise $\chi^{2}$ unfolding, Figure 1 (bottom).

In Figure 2, the reconstructed energy from channel 19 (top) and channel 47 (bottom) of the TileCal LBA23 module are shown in ADC counts before (red dots) and after (blue line) applying the $\chi^{2}$ unfolding. Channel 19 is an example of a non-correlated channel and the signal remains uncorrelated after applying the method. Channel 47 is an example of a highly correlated to its neighbors channel and the tails are significantly reduced after applying the method.

In Figure 3, the reconstructed energy from LBA23 channel 46 is plotted against the one from channel 47 before (top) and after (bottom) unfolding the correlated noise component with the $\chi^{2}$ method. A clear improvement is observed i.e., the correlation between both readout channels are very much reduced after applying the unfolding.
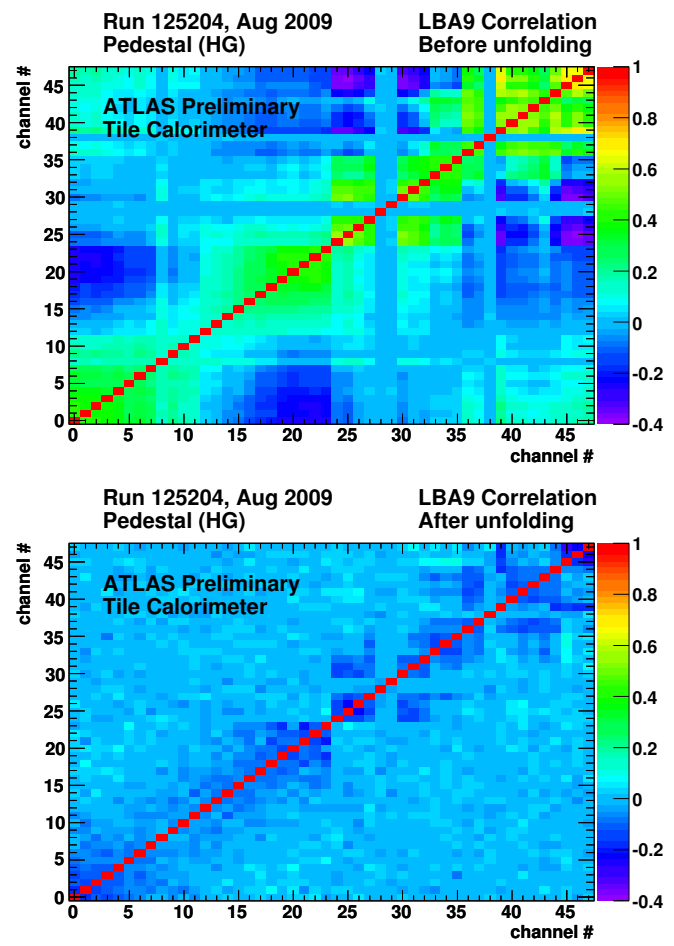

Fig. 1. Correlation plot of the noise value (evaluated as explained in the text) for all the channels in module LBA9 before (top) and after (bottom) the unfolding of the noise correlation effect.
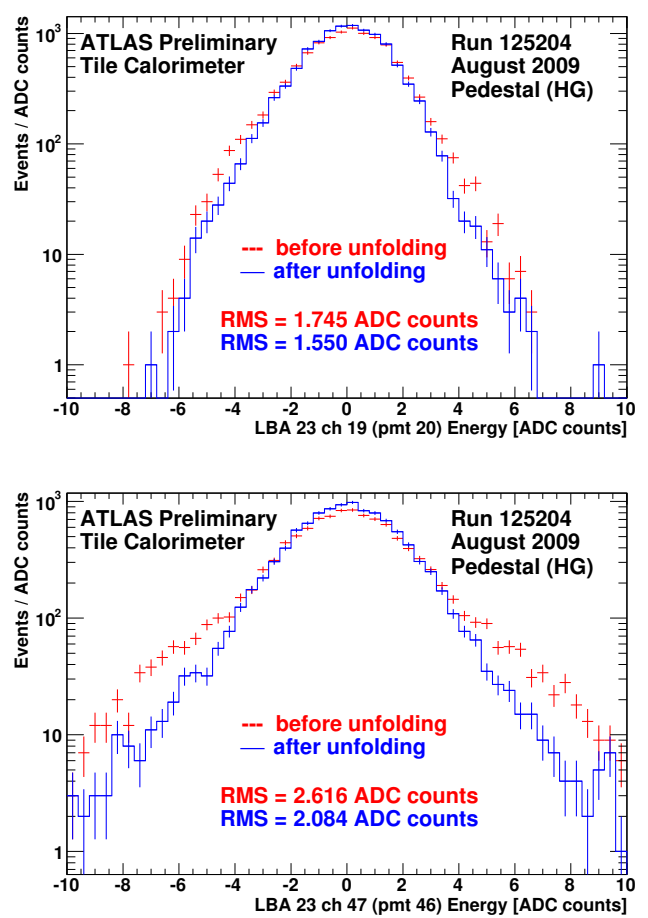

Fig. 2. LBA23 channels 19 (top) and 47 (bottom) before and after applying the $\chi^{2}$ unfolding. 


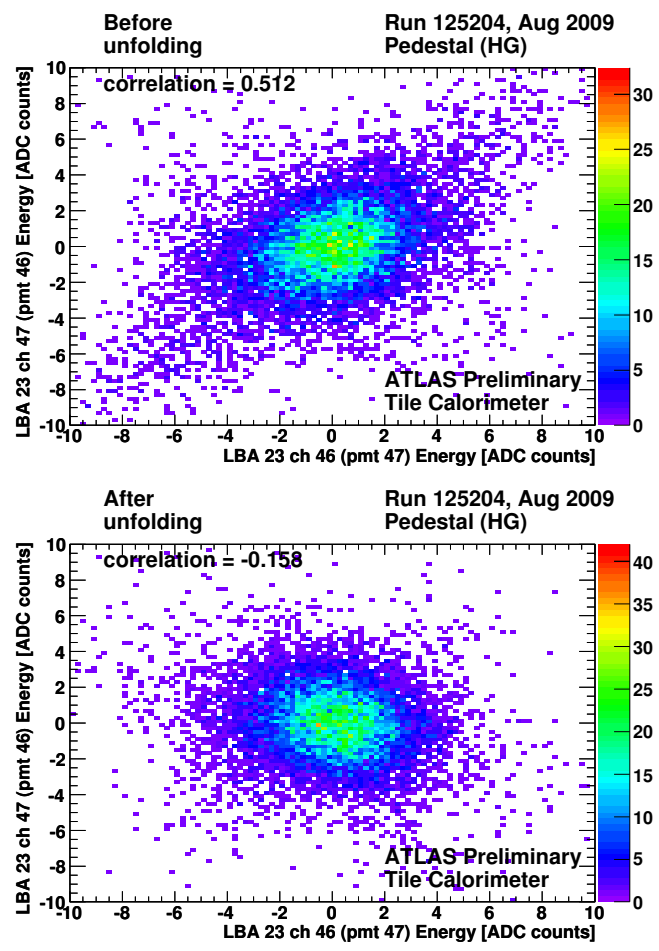

Fig. 3. LBA23 channel 46 against channel 47 before (top) and after (bottom) applying the $\chi^{2}$ unfolding.

\section{Calibration Run Analysis}

The correlation matrices calculated for the third ${ }^{1}$ of the seven digitized samples of a channel signal pulse are shown in Figure 4 before (top) and after (bottom) applying the correlated noise $\chi^{2}$ unfolding. A calibration run where a charge of 100 $\mathrm{pC}$ is injected, per event, by the Charge Injection System in each read-out channel of the drawer was used. A total of 1000 events per channel were analyzed when channel 3 of LBA48 was fired. The information from the first out of seven digitized samples (only sensitive to pedestal noise) is successfully used to unfold the noise correlations in the presence of physics signals. The correlated noise component is significantly reduced for third sample. The unfolding was applied to all samples with similar results to validate the performance of the unfolding method in the presence of a controlled injected signal.

\section{Method Performance with Physics Signals}

The validation of the method, in the presence of physics signals, is presented using a simulated sample with realistic correlations added and a collisions' data sample from Minimum Bias events. The information from the first out of seven digitized samples of a channel signal pulse (only sensitive to pedestal noise) is used to unfold the noise correlations in the presence of physics signals, only possible because no additional cross-talk was observed in the presence

\footnotetext{
${ }^{1}$ The peak of the pulse is timed to be between the third and the fourth samples.
}
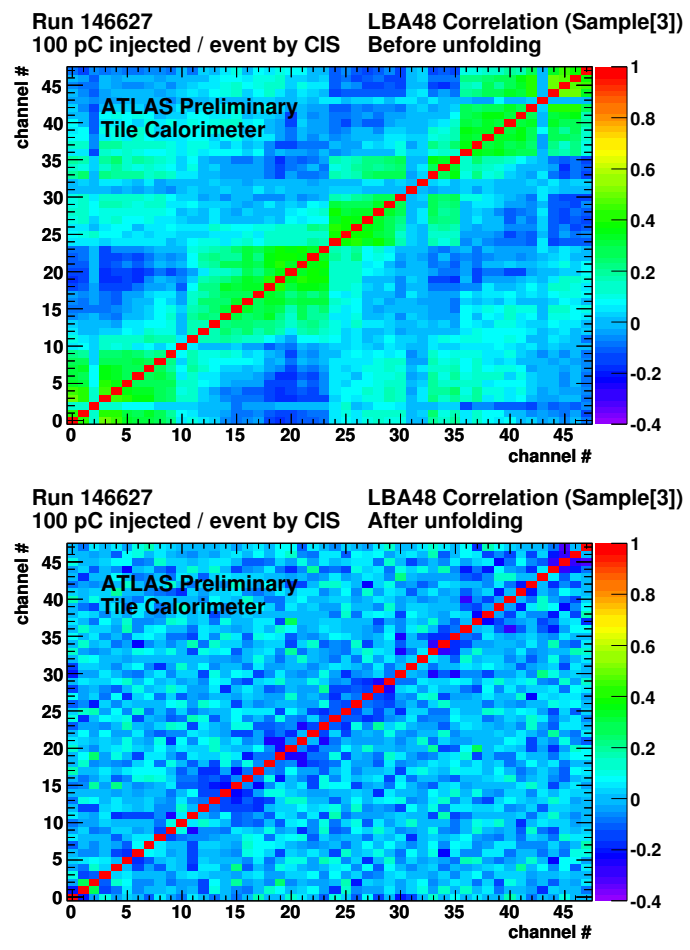

Fig. 4. Correlation matrices for LBA48 using the third out of seven digitized samples before (top) and after (bottom) applying the $\chi^{2}$ unfolding.

of controlled injected signals using special calibration runs. The reconstructed cell energy for 10,000 t $\bar{t}$ simulated events generated at $7 \mathrm{TeV}$ with MC@NLO [6] is presented in the Figure 5 (top) for three different situations: without channelto-channel correlations (black dotted line), with correlations before the noise correlations unfolding (red dashed line) and with correlations after noise correlations unfolding (blue full line). The reconstructed energy for Minimum Bias trigger data taken at a centre-of-mass energy of $900 \mathrm{GeV}$ [7] $(10,000$ events collected in December 2009) is shown in the Figure 5 (bottom). The red dashed line is before the noise correlations unfolding and the blue full line is after the unfolding. All readout channels were included in the figure and the signal region is shown for both. No visible bias is introduced by the noise correlations unfolding method. The correlations seen in the pedestal region can be successfully unfolded with the $\chi^{2}$ method without affecting the signal distribution.

\section{CONCLUSIONS}

The method proposed to remove the correlated noise component of the TileCal has been tested and approved through this systematic survey of the TileCal modules as a powerful solution to the coherent noise presence in pedestal runs. This approach shall be regarded as well as an effective diagnosis tool to the general behaviour of TileCal modules.

The analysis of special calibration runs, where only one channel is fired at a time, excluded the presence of crosstalk allowing the method described herein to be applied in the 

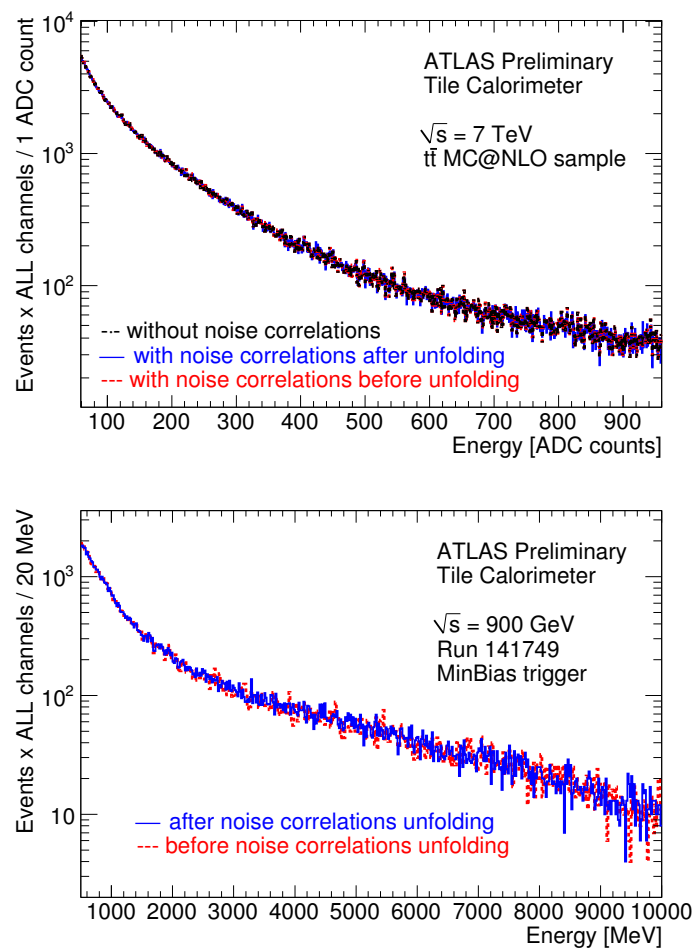

Fig. 5. Reconstructed energy for $t \bar{t}$ simulated events generated at $7 \mathrm{TeV}$ (top) and real $900 \mathrm{GeV}$ collisions data (bottom) before and after applying the $\chi^{2}$ unfolding.

presence of physics signals. No degradatation of the signal was observed. The results on Minimum Bias and simulated $t \bar{t}$ events show a clear improvement on the pedestal noise distribution and again the signal is not degraded as one would expect. In conclusion, the method proved to be very effective and reliable for different kinds of events, both real and simulated.

\section{ACKNOWLEDGMENT}

The work of M.C.N. Fiolhais has been supported by Fundação para a Ciência e Tecnologia (FCT), grant SFRH/BD/48680/2008.

\section{REFERENCES}

[1] G. Aad et al., ATLAS Collaboration, The ATLAS Experiment at the CERN Large Hadron Collider, JINST 3 S08003 (2008).

[2] ATLAS Tile Calorimeter Collaboration, Tile Calorimeter Technical Design Report, CERN/LHCC/96-42 (1996).

[3] F. Spanò, ATL-TILECAL-PUB-2008-011, 30 October 2008.

[4] P. Adragna et al., TileCal Collaboration, Testbeam studies of production modules of the ATLAS Tile Calorimeter, Nucl. Inst. Meth. A 606 (2009) 362394.

[5] G. Usai, ATL-TILECAL-PROC-2010-008, 13 August 2010.

[6] S. Frixione and B.R. Webber, Matching NLO QCD computations and parton shower simulations, JHEP 0206 (2002) 029 [hep-ph / 0204244$]$

[7] G. Aad et al., ATLAS Collaboration, Charged-particle multiplicities in pp interactions at sqrt(s) $=900 \mathrm{GeV}$ measured with the ATLAS detector at the LHC, Phys Lett B 688, 2010, Issue 1, 21-42. 